\title{
Enhancement of the room temperature luminescence of InAs quantum dots by GaSb capping
}

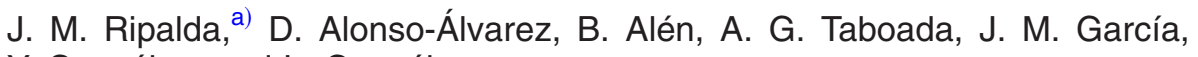 \\ Y. González, and L. González \\ Instituto de Microelectrónica de Madrid, CNM (CSIC), C/Isaac Newton 8, PTM, 28760 Tres Cantos, \\ Madrid, Spain
}

(Received 27 March 2007; accepted 8 June 2007; published online 5 July 2007)

\begin{abstract}
The authors have studied the use of antimony for the optimization of the InAs/GaAs(001) self-assembled quantum dot (QD) luminescence characteristics in the $1.3 \mu \mathrm{m}$ spectral region. The best results have been obtained by capping InAs QDs with $2 \mathrm{ML}$ of GaSb grown on top of a 3 ML GaAs barrier separating the InAs and the GaSb layers. This results in an order of magnitude enhancement of the room temperature luminescence intensity at $1.3 \mu \mathrm{m}$ emission wavelength. (C) 2007 American Institute of Physics. [DOI: 10.1063/1.2753716]
\end{abstract}

During the last decade, great progress has been achieved on the fabrication of optoelectronic devices based on selfassembled semiconductor nanostructures. ${ }^{1}$ Much of the work up to date has been based on InAs quantum dots (QDs) on GaAs substrates, but many other types of nanostructures have been extensively studied, such as InAs/GaAs quantum rings and InAs/InP quantum wires. ${ }^{2,3}$ More recently, nanostructures combining the electronic properties of more than two semiconductor compounds are being studied. Of particular interest is the $\mathrm{GaSb} / \mathrm{InAs} / \mathrm{GaAs}$ combination due to the type II band alignment expected between arsenides and antimonides. $^{4-6}$ Much of the recent research has been focused on reaching the long wavelengths of interest in optical fiber telecommunications (1300 and $1550 \mathrm{~nm}$ ) on GaAs substrates as an alternative to the more expensive technology based on InP substrates. ${ }^{4,5,7,8}$ In this letter, rather than extending the emission wavelength, we focus instead on maximizing the room temperature emission intensity of InAs QDs by adding antimony after QD nucleation.

Samples were grown by molecular beam epitaxy (MBE) on GaAs (001) substrates. All samples are combinations of four growth steps. Step 1 is InAs QD growth. QDs were observed by reflection high energy electron diffraction (RHEED) after deposition of $1.65 \mathrm{ML}$ of InAs at $510^{\circ} \mathrm{C}$ substrate temperature and at a $0.02 \mathrm{ML} / \mathrm{s}$ growth rate. The total InAs deposited was 2.6 ML. Step 2 is a $10 \mathrm{~s} \mathrm{Sb}$ exposure at a beam equivalent pressure of $1.5 \times 10^{-7}$ mbar. In our system this is roughly equivalent to $3 \mathrm{ML}$ of $\mathrm{Sb}$ in $\mathrm{GaSb}(001)$. Step 3 consists of the deposition of $3 \mathrm{ML}$ of GaAs. Step 4 consists of the deposition of $2 \mathrm{ML}$ of GaSb. The capping temperature $T_{c}$ during steps 2,3 , and 4 ranged from 510 to $470{ }^{\circ} \mathrm{C}$. Samples for optical characterization were then capped with an $80 \mathrm{~nm}$ thick GaAs layer. The temperature was held at $T_{c}$ until the first $10 \mathrm{~nm}$ of GaAs had been deposited, at which point the temperature was ramped up to $580{ }^{\circ} \mathrm{C}$. The $\mathrm{As}$ and $\mathrm{Sb}$ beam equivalent pressures were $1.7 \times 10^{-6}$ and $1.5 \times 10^{-7} \mathrm{mbar}$, respectively. In our MBE system, these values are, respectively, equivalent to $1.8 \times 10^{15}$ and $1.6 \times 10^{14}$ atoms $/ \mathrm{s} \mathrm{cm}^{2}$.

Figure 1 shows, for various growth sequences, the evolution of the integrated intensity of the (002) transmission

${ }^{a)}$ Electronic mail: ripalda@imm.cnm.csic.es diffraction spot due to the presence of QDs on the surface. A $10 \mathrm{~s}$ exposure to $\mathrm{Sb}$ considerably delays the decay of the QD diffraction signal during GaAs capping [Fig. 1(b)]. Capping with GaSb [Fig. 1(c)] first induces a decay of the QD signal, and after deposition of 1.5 ML induces a sharp increase of the QD transmission diffraction spot. This coverage is considerably smaller than the reported GaSb QD critical thickness on bare $\mathrm{GaAs}(001)$ surfaces.

The room temperature photoluminescence (PL) spectra corresponding to QDs with and without the GaSb layer are shown in Fig. 2. InAs QDs without any Sb content but prepared and characterized in the same conditions as the QDs with $\mathrm{Sb}$ capping are also shown for comparison. Postgrowth exposure of the InAs QD to antimony drastically increases the photoluminescence intensity and induces a $10 \mathrm{~nm}$ redshift (InAs + Sb curve in Fig. 2). The most plausible cause of such an increase in the room temperature emission intensity with $\mathrm{Sb}$ content is the increased valence band discontinuity due to $\mathrm{Sb}$ incorporation leading to an increased hole localization energy. Deposition of a $2 \mathrm{ML} \mathrm{GaSb}$ layer, with or without a $3 \mathrm{ML}$ GaAs barrier, leads to a shift of the emission wavelength to $1300 \mathrm{~nm}$ ( $\mathrm{InAs}+\mathrm{Sb}+\mathrm{GaAs}+\mathrm{GaSb}$ curve in

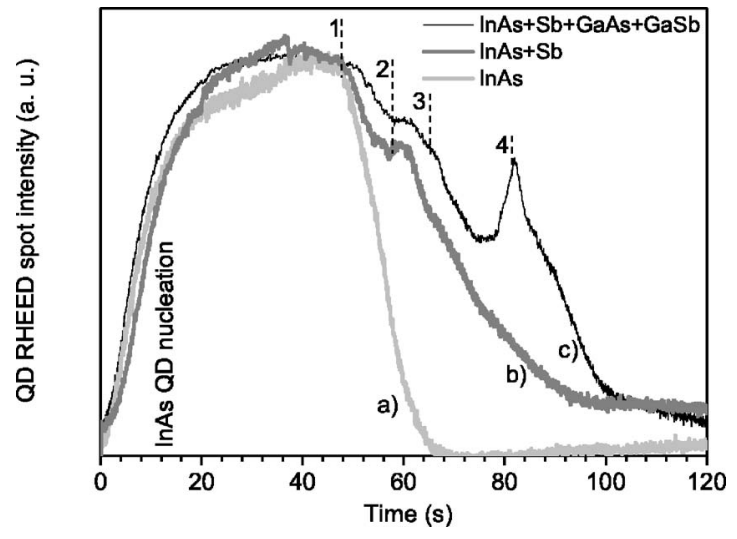

FIG. 1. Integrated RHEED intensity of the (002) transmission diffraction spot due to the presence of QDs on the surface for different growth sequences. (a) InAs QDs capped with GaAs. (b) InAs QDs exposed to Sb for $10 \mathrm{~s}$ and then capped with GaAs. (c) InAs QDs exposed to $\mathrm{Sb}$ for $10 \mathrm{~s}$ and then capped with $3 \mathrm{ML}$ of GaAs and $2 \mathrm{ML}$ of GaSb. The labels for steps 1, 2,3 , and 4 mark the end of each of the following layers: InAs, Sb exposure, 3 ML GaAs cap, and $2 \mathrm{ML} \mathrm{GaSb}$ cap. After the last step, all samples are capped with $80 \mathrm{~nm} \mathrm{GaAs}$ at $480{ }^{\circ} \mathrm{C}$. 


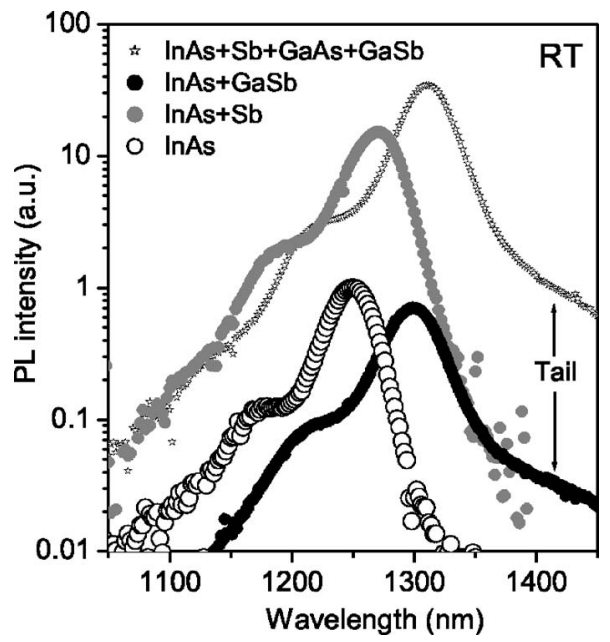

FIG. 2. Room temperature photoluminescence corresponding to (a) InAs QDs, (b) InAs QDs exposed to Sb, (c) InAs QDs capped with a 2 ML GaSb layer. (d) InAs QDs exposed to Sb, capped with a $3 \mathrm{ML} \mathrm{GaAs}$ barrier and then a $2 \mathrm{ML} \mathrm{GaSb}$ layer. The excitation power is $15 \mathrm{~mW}$ at $808 \mathrm{~nm}$.

Fig. 2). The sample with the GaSb layer deposited immediately on top of the InAs QDs (steps 1+4) has an emission intensity that is 50 times weaker than the sample that includes the GaAs spacer (steps $1+2+3+4$ ). This cannot be attributed to thermal escape, as in the case of InAs QDs, because the low temperature luminescence is also weak. The InAs QDs are close to the critical thickness for dislocation formation, and the extra stress induced by GaSb incorporation without the GaAs spacer layer might be leading to an increased density of defects and consequently lower intensity of the luminescence.

The higher emission intensity at all temperatures in the sample including a $3 \mathrm{ML} \mathrm{GaAs}$ spacer might be due to a smaller density of defects, or to a larger oscillator strength. Such thin GaAs capping layers are known to lead to a truncation of InAs QD height, ${ }^{10}$ and are often used as a method to tune the emission wavelength of QDs. ${ }^{11}$

A low energy tail ranging from 1350 to $1600 \mathrm{~nm}$ is observed only in those samples including a GaSb layer. We suspect such low energy tail to be due to type II spatially indirect recombinations of electrons in the InAs layer with holes confined in the GaSb layer. Such transitions are expected to be weaker than the type I spatially direct transitions due to the smaller overlap of the electron and hole wave functions. The nature of such low energy transitions is further elucidated by the power dependence of the low temperature PL spectra shown in Fig. 3. The low temperature PL spectra at $5 \mathrm{~mW}$ excitation power has a tail at energies below $950 \mathrm{meV}$ that saturates as the power is increased to $45 \mathrm{~mW}$. The long radiative lifetime of the states corresponding to type II indirect low energy emission might lead to these states quickly becoming filled as the excitation power increases, saturating the PL emission at energies below $950 \mathrm{meV}$. The separation between the ground state and the first excited state emission is $56 \mathrm{meV}$ for InAs QDs and $63 \mathrm{meV}$ for the InAs+Sb+GaAs+GaSb QDs. Typically, a larger excited state energy separation relative to the ground state emission is indicative of either a smaller QD volume, or a larger heterostructure band discontinuity, the later being a more likely possibility in our case. The energy difference between the second and third excited states is $61 \mathrm{meV}$ for the $\mathrm{Sb}$ capped QDs. The wetting layer peak (not shown) appears Downloaded 14 Apr 2009 to 161.111.235.57. Redistribution subject

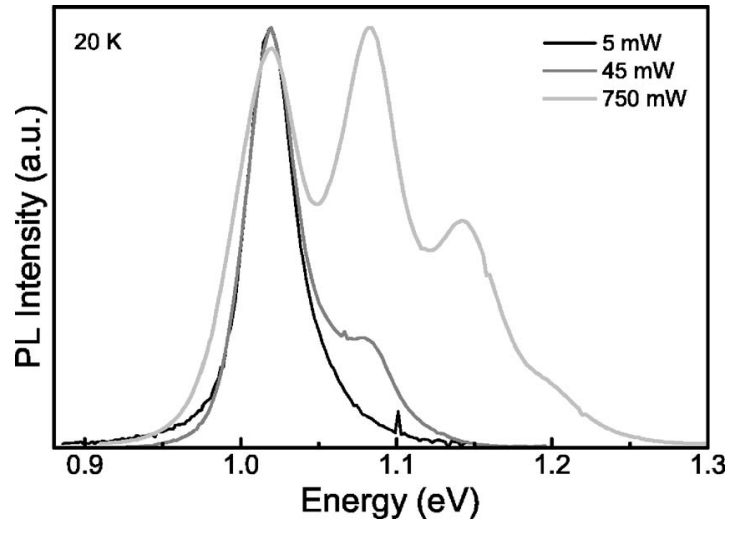

FIG. 3. Normalized low temperature PL spectra at different excitation powers for $\mathrm{InAs}+\mathrm{Sb}+\mathrm{GaAs}+\mathrm{GaSb}$ QDs.

at an unusually long wavelength $(1.22 \mathrm{eV}$ at room temperature, $1.29 \mathrm{eV}$ at $20 \mathrm{~K}$ ) possibly due to coupling or intermixing of the InAs and GaSb wetting layers.

The increased room temperature luminescence intensity in the $\mathrm{Sb}$ containing samples is due to a less pronounced decay of the luminescence as the temperature is increased. This is illustrated in Fig. 4, showing the temperature dependence of the integrated luminescence intensity for InAs QDs and InAs $+\mathrm{Sb}+\mathrm{GaAs}+\mathrm{GaSb}$ QDs. The luminescence of the $\mathrm{Sb}$ containing QDs reaches a maximum at $170 \mathrm{~K}$. This is a signature of carriers being trapped at low temperatures in states with long radiative lifetimes due to small electron-hole overlap, and then, as the temperature is raised, carriers are thermally excited to states with a larger electron-hole overlap. In our case this might be due to holes being trapped in the GaSb layer, were the valence band discontinuity is the largest. Electron-hole overlap in the GaSb layer is small as the electrons are repelled by the upward discontinuity in the conduction band. Most of the radiative recombination is expected to occur in the InAs layer, where there is a confinement potential for both electrons and holes. As the temperature rises, there is an increased likelihood of holes being thermally excited from the GaSb layer across the GaAs barrier to the InAs QDs. The fitting of the temperature dependencies in Fig. 4 to an Arrhenius-type equation in the high temperature range yields thermal escape barriers of 270 and $218 \mathrm{meV}$ for the samples with and without $\mathrm{Sb}$, respectively. The $52 \mathrm{meV}$ difference between these two thermal escape barriers nearly matches the measured $45 \mathrm{meV}$ redshift be-

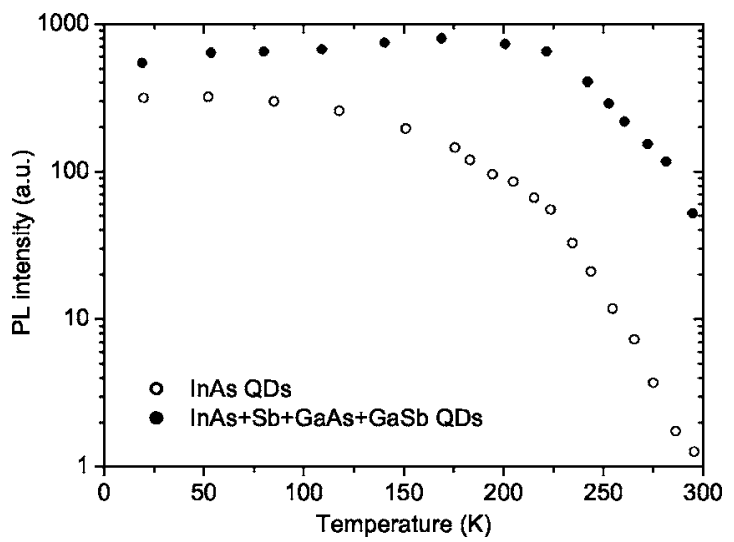

FIG. 4. Photoluminescence intensity as a function of temperature for InAs and $\mathrm{InAs}+\mathrm{Sb}+\mathrm{GaAs}+\mathrm{GaSb}$. The excitation power is $15 \mathrm{~mW}$ at $808 \mathrm{~nm}$. 


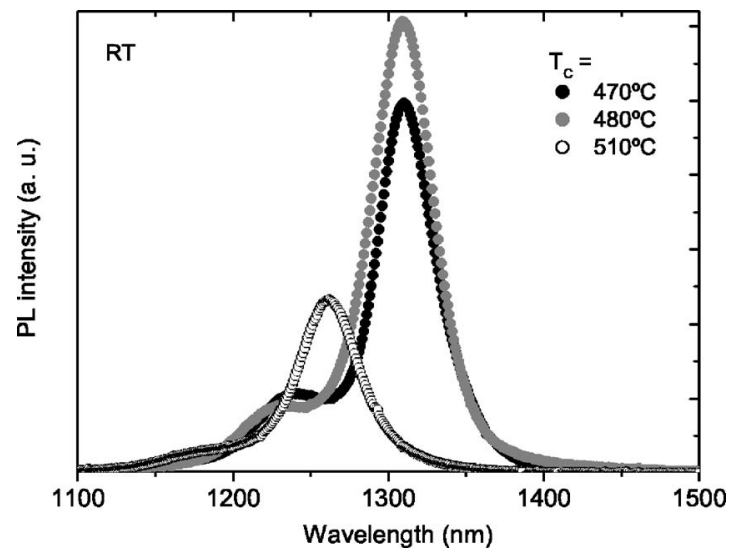

FIG. 5. Room temperature photoluminescence as a function of the $\mathrm{GaSb} / \mathrm{GaAs}$ capping temperature $T_{c}$.

tween these two samples. The increase of the thermal escape barrier with $\mathrm{Sb}$ incorporation suggests that the luminescence is limited by hole thermal escape, as $\mathrm{Sb}$ incorporation is expected to induce an upward shift in both the conduction and valence bands. ${ }^{12}$

It was found possible to further redshift the emission by increasing either the In or Sb content, but this led to a drastic decrease in emission intensity. This decrease of the emission intensity can be avoided by using thicker GaAs spacer layers, but then emission wavelengths shorter than $1300 \mathrm{~nm}$ are obtained. A crucial parameter was found to be the growth temperature $T_{c}$ used after the initial InAs QD layer. This was found to be optimal at $480{ }^{\circ} \mathrm{C}$, as shown in Fig. 5. Substrate temperatures during capping above $500{ }^{\circ} \mathrm{C}$ are known to lead to strong intermixing phenomena and a blueshift of the emission. ${ }^{13}$ Low substrate temperatures better preserve the QDs during capping, but the growth of GaAs at low temperatures also implies an increased density of point defects. Attempts to incorporate Sb during the InAs QD nucleation step resulted in a large dispersion of the QD size distribution, in agreement with the results by Kudo et al. ${ }^{14}$

Summarizing, it is shown that the optimized incorporation of Sb during capping of InAs QDs can lead to an order of magnitude improvement of the room temperature luminescence intensity in the $1.3 \mu \mathrm{m}$ spectral range. This might enable a drastic improvement of GaAs based optoelectronic devices for optical fiber telecommunications.

Support by CAM (Project Nos. 200560M089, S-505/ ENE-310, S-505/ESP/000200), by MEC (Project Nos. TEC2005-05781-C03-01, NAN2004-09109-C04-01, ConsoliderIngenio 2010 CSD2006-00019, RyC2003) and by the European Commission through the SANDIE Network of Excellence (NMP4-CT-2004-500101) is acknowledged. This work would have not been possible without the expert help of F. Briones and D. Fuster.

${ }^{1}$ D. L. Huffaker, G. Park, Z. Zou, O. B. Shchekin, and D. G. Deppe, Appl. Phys. Lett. 73, 2564 (1998).

${ }^{2}$ F. Suárez, D. Granados, M. L. Dotor, and J. M. García, Nanotechnology 15, S126 (2004).

${ }^{3}$ D. Fuster, M. U. González, L. González, Y. González, T. Ben, A. Ponce, S. Molina, and J. Martínez-Pastor, Appl. Phys. Lett. 85, 1424 (2004).

${ }^{4}$ H. Y. Liu, M. J. Steer, T. J. Badcock, D. J. Mowbray, M. S. Skolnick, F. Suarez, J. S. Ng, M. Hopkinson, and J. P. R. David, J. Appl. Phys. 99, 046104 (2006).

${ }^{5}$ J. M. Ripalda, D. Granados, Y. González, A. M. Sánchez, S. I. Molina, and J. M. García, Appl. Phys. Lett. 87, 202108 (2005).

${ }^{6}$ T. Matsuura, T. Miyamoto, and F. Koyama, Appl. Phys. Lett. 88, 183109 (2006).

${ }^{7}$ H. Y. Liu, M. J. Steer, T. J. Badcock, D. J. Mowbray, M. S. Skolnick, P. Navaretti, K. M. Groom, M. Hopkinson, and R. A. Hogg, Appl. Phys. Lett. 86, 143108 (2005).

${ }^{8}$ D. Guimard, S. Tsukamoto, M. Nishioka, and Y. Arakawa, Appl. Phys. Lett. 89, 083116 (2006).

${ }^{9}$ M. Geller, C. Kapteyn, L. Müller-Kirsch, R. Heitz, and D. Bimberg, Appl. Phys. Lett. 82, 2706 (2003).

${ }^{10}$ G. Costantini, A. Rastelli, C. Manzano, P. Acosta-Diaz, R. Songmuang, G. Katsaros, O. G. Schmidt, and K. Kern, Phys. Rev. Lett. 96, 226106 (2006).

${ }^{11}$ J. M. García, G. Medeiros-Ribeiro, K. Schmidt, T. Ngo, J. L. Feng, A. Lorke, J. Kotthaus, and P. M. Petroff, Appl. Phys. Lett. 71, 2014 (1997).

${ }^{12}$ C. E. Pryor and M. E. Pistol, Phys. Rev. B 72, 205311 (2005).

${ }^{13}$ E. C. Le Ru, P. Howe, T. S. Jones, and R. Murray, Phys. Rev. B 67, 165303 (2003).

${ }^{14}$ M. Kudo, T. Nakaoka, S. Iwamoto, and Y. Arakawa, Jpn. J. Appl. Phys., Part 2 44, L45 (2005). 